Article

\title{
Capture Effect in the FSA-Based Networks under Rayleigh, Rician and Nakagami- $m$ Fading Channels
}

\author{
Yang Wang ${ }^{(\mathbb{D}}$, Jianghong Shi and Lingyu Chen * \\ School of Information Science and Engineering, Xiamen University, Xiamen 361005, China; \\ yangwang@stu.xmu.edu.cn (Y.W.); shijh@xmu.edu.cn (J.S.) \\ * Correspondence: chenly@xmu.edu.cn; Tel.: +86-139-5008-3402
}

Received: 30 January 2018; Accepted: 7 March 2018; Published: 11 March 2018

\begin{abstract}
Framed slotted aloha (FSA) is a multiple access protocol widely used in wireless communication for its simplicity and effectivity. The theoretical maximum channel utilization of FSA is approximately equal to 0.37 irrespective of the capture effect. In fact, the capture effect is a common phenomenon in wireless communication, which can increase the channel utilization. In this paper, we derive the closed-form expressions of capture probabilities under Rayleigh, Rician and Nakagami- $m$ fading channels, respectively, and further give the optimal frame length which maximizes the channel utilization in the FSA-based networks. The numerical results are given for indicating the capture probabilities under different fading channels and their impacts on the optimal frame length and the maximum channel utilization.
\end{abstract}

Keywords: FSA; capture effect; Rayleigh; Rician; Nakagami-m; frame length; channel utilization

\section{Introduction}

Aloha type protocols, including slotted aloha and unslotted aloha, play an important role in the multiple access communication [1-5]. Framed slotted aloha (FSA), an improvement to the slotted aloha protocol, has been widely used in the satellite networks [6,7], radio frequency identification networks [8-11], wireless sensor networks [12,13], vehicular ad hoc networks [14-16], wireless cellular networks $[17,18]$, optical networks $[19,20]$ and so on. The reason is that FSA protocol is easy to realize in real communication systems and improves the transmission efficiency. In the FSA-based networks, the link-time is comprised of frames and each frame is further divided into slots. The number of slots within a frame can be fixed or even vary dynamically frame by frame on the basis of network condition. All the users in the network are frame- and slot-synchronized, and each user randomly chooses a slot of one frame for transmitting its packet. When a slot is chosen concurrently by two or more users, we usually consider it a collided slot. For this model of collision channel, the maximum channel utilization is approximately equal to $\frac{1}{e} \approx 0.37$ [21]. As a matter of fact, the kind of collided slot is likely to be a successful slot when the power level of one user is greater enough than that of the others'. The phenomenon is called capture effect, which often occurs in reality and improves the channel utilization of FSA-based networks.

In recent years, much attention has been paid to the capture effect in the FSA-based networks. Actually, though some literatures have indicated that the capture effect cannot be ignored, they usually assume that the probability of capture effect is a constant for simplicity or a variable without regard to the real channel condition [9-11,22,23]. Obviously, they do not clearly illustrate the relationships between the capture probabilities of different fading channels and the maximum channel utilization in the FSA-based networks, such as Rayleigh, Rician, and Nakagami- $m$ fading channel. Motivated by it, we analyze the capture effect in the FSA-based networks under different fading channels mentioned above. Since the setting of optimal frame length is the key to achieve the maximum channel utilization 
in the FSA-based networks, we give the optimal frame length with taking the capture effect into account. By means of setting the optimal frame length, the actual channel utilization is maximized.

The rest of this paper is organized as follows. In Section 2, the closed-form expressions of capture probability under Rayleigh, Rician and Nakagami- $m$ fading channels are derived, respectively. Section 3 indicates the relationship between the optimal frame length and the maximum channel utilization. The numerical results are given in Section 4 . Finally, Section 5 concludes the paper.

\section{Capture Probabilities Under Different Fading Channels}

The capture effect is a common phenomenon in wireless networks. There are two models for analyzing the capture effect [24-26]. In the first model, the capture effect occurs when the received signal power which comes from one transmitter is greater than each of the other interferer's power by a minimum threshold factor $z$, which is called the capture ratio. In the second model, one signal is captured by the receiver when its power level exceeds the sum of the power of all the other interfering signals by capture ratio $z$. Since the second model resembles more accurately the way a real receiver operates [27], we will adopt it to evaluate the capture probabilities under Rayleigh, Rician and Nakagami- $m$ fading channels, respectively.

For the sake of analysis, we disregard the effect of propagation path loss and assume that all signals transmitted have same average power value $\bar{\gamma}[25,28]$. We consider $n$ (an integer greater than one) packets transmitted simultaneously, which means that they choose the same slot for transmission. Among them, one is a test packet $t$ and the other $n-1$ packets are interfering packets. The condition for capture is $\frac{\gamma_{t}}{\sum_{k=1}^{n-1} \gamma_{k}}>z$, and the capture probability is given by $[25,28]$

$$
\begin{aligned}
p_{\text {cap }} & =n \int_{0}^{\infty} f_{\gamma_{t}}\left(\gamma_{t}\right) \cdot \operatorname{Pr}\left[\frac{\gamma_{t}}{\sum_{k=1}^{n-1} \gamma_{k}}>z\right] d \gamma_{t} \\
& =n \int_{0}^{\infty} f_{\gamma_{t}}\left(\gamma_{t}\right)\left[\int_{0}^{\frac{\gamma_{t}}{z}} g_{\gamma_{n-1}}\left(\gamma_{n-1}\right) d \gamma_{n-1}\right] d \gamma_{t},
\end{aligned}
$$

where $f_{\gamma_{t}}\left(\gamma_{t}\right)$ is the probability density function (PDF) of received power, and $g_{\gamma_{n-1}}\left(\gamma_{n-1}\right)$ is the compound PDF that result from the convolution of $n-1$ PDFs of received power.

Next, we will derive the expressions of capture probability under Rayleigh, Rician and Nakagami- $m$ fading channels, respectively.

\subsection{Rayleigh Fading Channel}

The Rayleigh fading channel is frequently used to model multi-path fading with no direct line-of-sight (LOS) path. The instantaneous received power in Rayleigh channel is given by [29]

$$
f_{\gamma}(\gamma)=\frac{1}{\bar{\gamma}} e^{-\frac{\gamma}{\bar{\gamma}}}, \gamma \geq 0
$$

According to the theorem of Laplace transform, we can obtain

$$
f_{\gamma}(\gamma) \leftrightarrow F_{\gamma}(s)=\frac{1}{\bar{\gamma}} \frac{1}{s+\frac{1}{\bar{\gamma}}} .
$$

To do the convolution of $n-1$ independent and identically distributed (i.i.d.) signals, we apply the property of Laplace transform. Then, we have

$$
f_{\gamma}(\gamma)^{\otimes n-1} \leftrightarrow G_{\gamma_{n-1}}(s)=\frac{1}{\bar{\gamma}^{n-1}} \frac{1}{\left(s+\frac{1}{\bar{\gamma}}\right)^{n-1}} .
$$


By applying the inverse Laplace transformation, we can obtain

$$
G_{\gamma_{n-1}}(s) \leftrightarrow g_{\gamma_{n-1}}\left(\gamma_{n-1}\right)=\frac{\gamma_{n-1}^{n-2}}{\bar{\gamma}^{n-1}(n-2) !} e^{-\frac{\gamma_{n-1}}{\gamma}}
$$

After substituting (2) and (5) into (1), the capture probability in Rayleigh fading channel can be written as

$$
\begin{aligned}
p_{\text {cap }}^{\text {Ray }} & =n \int_{0}^{\infty} \frac{1}{\bar{\gamma}} e^{-\frac{\gamma_{t}}{\bar{\gamma}}}\left[\int_{0}^{\frac{\gamma_{t}}{z}} \frac{\gamma_{n-1}^{n-2}}{\bar{\gamma}^{n-1}(n-2) !} e^{-\frac{\gamma_{n-1}}{\gamma}} d \gamma_{n-1}\right] d \gamma_{t} \\
& =\frac{n}{\bar{\gamma}^{n}(n-2) !} \int_{0}^{\infty} e^{-\frac{\gamma_{t}}{\gamma}}\left[\int_{0}^{\frac{\gamma_{t}}{z}} \gamma_{n-1}^{n-2} e^{-\frac{\gamma_{n-1}}{\gamma}} d \gamma_{n-1}\right] d \gamma_{t}
\end{aligned}
$$

According to [30] (p. 900), i.e.,

$$
\gamma(k, x)=(k-1) !\left[1-e^{-x} \sum_{j=0}^{k-1} \frac{x^{j}}{j !}\right], k=1,2, \ldots
$$

where $\gamma(\cdot, \cdot)$ is the lower incomplete gamma function defined as $\gamma(\alpha, x)=\int_{0}^{\infty} y^{\alpha-1} e^{-y} d y$, we have

$$
\begin{aligned}
\int_{0}^{\frac{\gamma_{t}}{z}} \gamma_{n-1}^{n-2} e^{-\frac{\gamma_{n-1}}{\bar{\gamma}} d \gamma_{n-1}} & =\bar{\gamma}^{n-1} \int_{0}^{\frac{\gamma_{t}}{z \bar{\gamma}}}\left(\frac{\gamma_{n-1}}{\bar{\gamma}}\right)^{n-2} e^{-\frac{\gamma_{n-1}}{\bar{\gamma}}} d\left(\frac{\gamma_{n-1}}{\bar{\gamma}}\right) \\
& =\bar{\gamma}^{n-1} \gamma\left(n-1, \frac{\gamma_{t}}{z \bar{\gamma}}\right) \\
& =\bar{\gamma}^{n-1}(n-2) !\left[1-e^{-\frac{\gamma_{t}}{z \bar{\gamma}}} \sum_{k=0}^{n-2} \frac{\left(\frac{\gamma_{t}}{z \bar{\gamma}}\right)^{k}}{k !}\right] .
\end{aligned}
$$

After substituting (8) into (6), we can obtain

$$
\begin{aligned}
p_{\text {cap }}^{\text {Ray }} & =n\left[\frac{1}{\bar{\gamma}} \int_{0}^{\infty} e^{-\frac{\gamma_{t}}{\gamma}} d \gamma_{t}-\frac{1}{\bar{\gamma}} \int_{0}^{\infty} e^{-\frac{\gamma_{t}}{\bar{\gamma}} \frac{z+1}{z}} \sum_{k=0}^{n-2} \frac{\left(\frac{\gamma_{t}}{z \bar{\gamma}}\right)^{k}}{k !} d \gamma_{t}\right] \\
& =n\left[1-\frac{1}{\bar{\gamma}} \int_{0}^{\infty} e^{-(z+1) \frac{\gamma_{t}}{z \bar{\gamma}}} \sum_{k=0}^{n-2} \frac{\left(\frac{\gamma_{t}}{z \bar{\gamma}}\right)^{k}}{k !} d \gamma_{t}\right] .
\end{aligned}
$$

Let $\frac{\gamma t}{z \bar{\gamma}}=u$ and then

$$
\begin{aligned}
\frac{1}{\bar{\gamma}} \int_{0}^{\infty} e^{-(z+1) \frac{\gamma t}{z \bar{\gamma}} \sum_{k=0}^{n-2} \frac{\left(\frac{\gamma t}{z \bar{\gamma}}\right)^{k}}{k !} d \gamma_{t}} & =z\left[\int_{0}^{\infty} e^{-(z+1) u} d u+\int_{0}^{\infty} e^{-(z+1) u} u d u+\cdots\right. \\
& \left.+\int_{0}^{\infty} e^{-(z+1) u} \frac{u^{n-2}}{(n-2) !} d u\right] \\
& =z\left[\frac{1}{z+1}+\frac{1}{(z+1)^{2}}+\cdots+\frac{1}{(z+1)^{n-1}}\right] \\
& =z \sum_{k=0}^{n-2} \frac{1}{(z+1)^{k+1}} .
\end{aligned}
$$


Then, substituting (10) into (9), the capture probability can be written as

$$
p_{\text {cap }}^{\text {Ray }}=n\left(1-z \sum_{k=0}^{n-2} \frac{1}{(z+1)^{k+1}}\right)
$$

\subsection{Rician Fading Channel}

The instantaneous received power in Rician fading channel is given by [31]

$$
f_{\gamma}(\gamma)=\frac{1+K}{\bar{\gamma}} e^{-K-\frac{\gamma(1+K)}{\gamma}} I_{0}\left(2 \sqrt{\frac{K(1+K) \gamma}{\bar{\gamma}}}\right), \gamma \geq 0,
$$

where the factor $K$ corresponds to the ratio of the power of LOS component to the average power of scattered components and $I_{0}(\cdot)$ is the modified Bessel function of the first kind, whose general form is given by

$$
I_{\alpha}(x)=\sum_{k=0}^{\infty} \frac{1}{k ! \Gamma(k+\alpha+1)}\left(\frac{x}{2}\right)^{2 k+\alpha}
$$

where $\Gamma(\cdot)$ is the gamma function defined as $\Gamma(\alpha)=\int_{0}^{\infty} x^{\alpha-1} e^{-x} d x$. According to [32] (p. 1026), i.e.,

$$
\left(\frac{x}{\beta}\right)^{\frac{\mu-1}{2}} I_{\mu-1}(2 \sqrt{\beta x}) \leftrightarrow \frac{1}{s^{\mu}} e^{\frac{\beta}{s}}
$$

Let $\mu=0, \beta=\frac{K(1+K)}{\bar{\gamma}}$ in (14) and apply the shifting property, we can obtain

$$
f_{\gamma}(\gamma) \leftrightarrow F_{\gamma}(s)=\frac{1+K}{\bar{\gamma}} e^{-K} \frac{1}{s+\frac{1+K}{\bar{\gamma}}} \exp \left[\frac{\frac{K(1+K)}{\bar{\gamma}}}{s+\frac{1+K}{\bar{\gamma}}}\right] .
$$

According to the convolution property of Laplace transform, the following expression can be easily obtained

$$
f_{\gamma}(\gamma)^{\otimes n-1} \leftrightarrow G_{\gamma_{n-1}}(s)=\left(\frac{1+K}{\bar{\gamma}}\right)^{n-1} e^{-(n-1) K}\left[\frac{1}{s+\frac{1+K}{\bar{\gamma}}}\right]^{n-1} \exp \left[\frac{\frac{(n-1) K(1+K)}{\bar{\gamma}}}{s+\frac{1+K}{\bar{\gamma}}}\right] .
$$

Taking advantage of the inverse Laplace transformation, (14) and [30] (p. 26), i.e., $e^{x}=\sum_{k=0}^{\infty} \frac{x^{k}}{k !}$, we can obtain

$$
\begin{aligned}
& G_{\gamma_{n-1}}(s) \leftrightarrow g_{\gamma_{n-1}}\left(\gamma_{n-1}\right)=\left(\frac{1+K}{\bar{\gamma}}\right)^{\frac{n}{2}} {\left[\frac{\gamma_{n-1}}{(n-1) K}\right]^{\frac{n}{2}-1} e^{-(n-1) K-\frac{\gamma_{n-1}(1+K)}{\bar{\gamma}}} } \\
& \cdot I_{n-2}\left(2 \sqrt{\frac{K(1+K)(n-1) \gamma_{n-1}}{\bar{\gamma}}}\right) .
\end{aligned}
$$

Then, we substitute (12), (17) into (1) and utilize (13), (7) and [30] (p. 900), i.e.,

$$
\Gamma(k, x)=(k-1) ! e^{-x} \sum_{j=0}^{k-1} \frac{x^{j}}{j !}, k=1,2, \ldots
$$


Let $\frac{1+K}{\tilde{\gamma}}=a$ for simplifying the writing, the capture probability in Rician fading channel can be written as

$$
\begin{aligned}
p_{c a p}^{R i c} & =n \int_{0}^{\infty} a e^{-K-a \gamma_{t}} I_{0}\left(2 \sqrt{a K \gamma_{t}}\right)\left\{\int_{0}^{\frac{\gamma_{t}}{z}} a^{\frac{n}{2}}\left[\frac{\gamma_{n-1}}{(n-1) K}\right]^{\frac{n}{2}-1}\right. \\
& \left.\cdot e^{-(n-1) K-a \gamma_{n-1}} I_{n-2}\left(2 \sqrt{a(n-1) K \gamma_{n-1}}\right) d \gamma_{n-1}\right\} d \gamma_{t} \\
& =n \int_{0}^{\infty} a e^{-K-a \gamma_{t}} \sum_{j=0}^{\infty} \frac{\left(a K \gamma_{t}\right)^{j}}{(j !)^{2}}\left\{1-e^{-(n-1) K-\frac{a \gamma_{t}}{z}} \sum_{i=0}^{\infty} \frac{[(n-1) K]^{i}}{i !} \sum_{k=0}^{n-2+i} \frac{\left(\frac{a \gamma_{t}}{z}\right)^{k}}{k !}\right\} d \gamma_{t} \\
& =n\left\{\int_{0}^{\infty} a e^{-K-a \gamma_{t}} \sum_{j=0}^{\infty} \frac{\left(a K \gamma_{t}\right)^{j}}{(j !)^{2}} d \gamma_{t}\right. \\
& \left.-\int_{0}^{\infty} a e^{-n K-\frac{a \gamma_{t}(z+1)}{z}} \sum_{j=0}^{\infty} \frac{\left(a K \gamma_{t}\right)^{j}}{(j !)^{2}} \sum_{i=0}^{\infty} \frac{[(n-1) K]^{i}}{i !} \sum_{k=0}^{n-2+i} \frac{\left(\frac{a \gamma_{t}}{z}\right)^{k}}{k !} d \gamma_{t}\right\}
\end{aligned}
$$

Next, we calculate the two parts in the brace of (19), respectively. Let $a \gamma_{t}=x$, the first part can be written as

$$
\begin{aligned}
\int_{0}^{\infty} a e^{-K-a \gamma_{t}} \sum_{j=0}^{\infty} \frac{\left(a K \gamma_{t}\right)^{j}}{(j !)^{2}} d \gamma_{t} & =e^{-K}\left[\int_{0}^{\infty} e^{-x} d x+K \int_{0}^{\infty} x e^{-x} d x+\frac{K^{2}}{(2 !)^{2}} \int_{0}^{\infty} x^{2} e^{-x} d x+\cdots\right] \\
& =e^{-K}\left[\Gamma(1)+K \Gamma(2)+\frac{K^{2}}{(2 !)^{2}} \Gamma(3)+\cdots\right] \\
& =e^{-K} \sum_{j=0}^{\infty} \frac{K^{j}}{j !}=e^{-K} e^{K}=1
\end{aligned}
$$

Let $\frac{a \gamma_{t}(z+1)}{z}=y$, the second part in the brace can be written as

$$
\begin{aligned}
& \int_{0}^{\infty} a e^{-n K-\frac{a \gamma_{t}(z+1)}{z}} \sum_{j=0}^{\infty} \frac{\left(a K \gamma_{t}\right)^{j}}{(j !)^{2}} \sum_{i=0}^{\infty} \frac{[(n-1) K]^{i}}{i !} \sum_{k=0}^{n-2+i} \frac{\left(\frac{a \gamma_{t}}{z}\right)^{k}}{k !} d \gamma_{t} \\
& =\frac{z}{z+1} e^{-n K} \sum_{i=0}^{\infty} \frac{[(n-1) K]^{i}}{i !}\left[\int_{0}^{\infty} e^{-y} \sum_{j=0}^{\infty} \frac{K^{j} y}{(j !)^{2}}\left(\frac{z}{z+1}\right)^{j} d y+(z+1)^{-1}\right. \\
& \left.\cdot \int_{0}^{\infty} y e^{-y} \sum_{j=0}^{\infty} \frac{K^{j} y}{(j !)^{2}}\left(\frac{z}{z+1}\right)^{j} d y+\cdots+\frac{(z+1)^{-(n-2+i)}}{(n-2+i) !} \int_{0}^{\infty} y^{n-2+i} e^{-y} \sum_{j=0}^{\infty} \frac{K^{j} y}{(j !)^{j}}\left(\frac{z}{z+1}\right)^{j} d y\right] \\
& =\frac{z}{z+1} e^{-n K} \sum_{i=0}^{\infty} \frac{[(n-1) K]^{i}}{i !}\left[\sum_{j=0}^{\infty} \frac{K^{j} j !}{(j !)^{2}}\left(\frac{z}{z+1}\right)^{j}+(z+1)^{-1} \sum_{j=0}^{\infty} \frac{K^{j}(j+1) !}{(j !)^{2}}\left(\frac{z}{z+1}\right)^{j}+\cdots\right. \\
& \left.+\frac{(z+1)^{-(n-2+i)}}{(n-2+i) !} \sum_{j=0}^{\infty} \frac{K^{j}(j+n-2+i) !}{(j !)^{2}}\left(\frac{z}{z+1}\right)^{j}\right] \\
& =e^{-n K} \sum_{i=0}^{\infty} \frac{[(n-1) K]^{i}}{i !} \sum_{k=0}^{n-2+i} \frac{\left(\frac{1}{z+1}\right)^{k}}{k !} \sum_{j=0}^{\infty} \frac{K^{j}(j+k) !}{(j !)^{2}}\left(\frac{z}{z+1}\right)^{j+1}
\end{aligned}
$$

Substituting (20) and (21) into (19), we can obtain the capture probability

$$
p_{c a p}^{R i c}=n\left\{1-e^{-n K} \sum_{i=0}^{\infty} \frac{[(n-1) K]^{i}}{i !} \sum_{k=0}^{n-2+i} \frac{\left(\frac{1}{z+1}\right)^{k}}{k !} \sum_{j=0}^{\infty} \frac{K^{j}(j+k) !}{(j !)^{2}}\left(\frac{z}{z+1}\right)^{j+1}\right\}
$$


The similar expression also can be found in $[25,28]$. Although the Rayleigh PDF (Equation (2)) can be obtained by Rician PDF (Equation (12)) with $K=0$, we cannot let $K=0$ in Equation (22). The reason is $K$ cannot be zero in Equation (17). That is to say, we derive the expression of capture probability in Rician fading channel based on the assumption $K>0$.

\subsection{Nakagami-m Fading Channel}

The instantaneous received power in Nakagami- $m$ fading channel is given by [33]

$$
f_{\gamma}(\gamma)=\frac{m^{m} \gamma^{m-1}}{\bar{\gamma}^{m} \Gamma(m)} e^{-\frac{m \gamma}{\gamma}}, \gamma \geq 0
$$

where $m$ is the shape parameter, which ranges from $\frac{1}{2}$ to $\infty$. By adopting the Laplace transform method again, we have

$$
f_{\gamma}(\gamma) \leftrightarrow F_{\gamma}(s)=\frac{m^{m}}{\bar{\gamma}^{m}} \frac{1}{\left(s+\frac{m}{\bar{\gamma}}\right)^{m}}
$$

Then, by making use of the convolution property of Laplace transform again, we can easily obtain

$$
f_{\gamma}(\gamma)^{\otimes n-1} \leftrightarrow G_{\gamma_{n-1}}(s)=\frac{m^{m(n-1)}}{\bar{\gamma}^{m(n-1)}} \frac{1}{\left(s+\frac{m}{\bar{\gamma}}\right)^{m(n-1)}}
$$

Then, we apply the inverse Laplace transformation again and have

$$
G_{\gamma_{n-1}}(s) \leftrightarrow g \gamma_{n-1}\left(\gamma_{n-1}\right)=\frac{m^{m(n-1)}}{\bar{\gamma}^{m(n-1)} \Gamma(m n-m)} \gamma_{n-1}^{m(n-1)-1} e^{-\frac{m \gamma_{n-1}}{\bar{\gamma}}}
$$

To simplify the writing, we let $\frac{m}{\bar{\gamma}}=a$, and then the capture probability in Nakagami- $m$ fading channel can be written as

$$
\begin{aligned}
p_{c a p}^{N a k} & =n \int_{0}^{\infty} \frac{a^{m} \gamma_{t}^{m-1}}{\Gamma(m)} e^{-a \gamma_{t}}\left[\int_{0}^{\frac{\gamma_{t}}{z}} \frac{a^{m(n-1)}}{\Gamma(m n-m)} \gamma_{n-1}^{m(n-1)-1} e^{-a \gamma_{n-1}} d \gamma_{n-1}\right] d \gamma_{t} \\
& =\frac{n a^{m n}}{\Gamma(m) \Gamma(m n-m)} \int_{0}^{\infty} \gamma_{t}^{m-1} e^{-a \gamma_{t}}\left[\int_{0}^{\frac{\gamma_{t}}{z}} \gamma_{n-1}^{m n-m-1} e^{-a \gamma_{n-1}} d \gamma_{n-1}\right] d \gamma_{t}
\end{aligned}
$$

According to [30] (p. 900), i.e.,

$$
\gamma(\alpha, x)=\sum_{k=0}^{\infty} \frac{(-1)^{k} x^{\alpha+k}}{k !(\alpha+k)}
$$

we can obtain

$$
\begin{aligned}
\int_{0}^{\frac{\gamma_{t}}{z}} \gamma_{n-1}^{m n-m-1} e^{-a \gamma_{n-1} d \gamma_{n-1}} & =\frac{1}{a^{m n-m}} \gamma\left(m n-m, \frac{a \gamma_{t}}{z}\right) \\
& =\frac{1}{a^{m n-m}} \sum_{k=0}^{\infty} \frac{(-1)^{k}\left(\frac{a \gamma_{t}}{z}\right)^{m n-m+k}}{k !(m n-m+k)}
\end{aligned}
$$

Substituting (29) into (27), we can obtain 


$$
\begin{aligned}
p_{c a p}^{N a k} & =\frac{n a^{m}}{\Gamma(m) \Gamma(m n-m)} \int_{0}^{\infty} \gamma_{t}^{m-1} e^{-a \gamma_{t}} \sum_{k=0}^{\infty} \frac{(-1)^{k}\left(\frac{a \gamma_{t}}{z}\right)^{m n-m+k}}{k !(m n-m+k)} d \gamma_{t} \\
& =\frac{n a^{m}}{\Gamma(m) \Gamma(m n-m)}\left[\int_{0}^{\infty} \gamma_{t}^{m-1} e^{-a \gamma_{t}} \frac{\left(\frac{a \gamma_{t}}{z}\right)^{m n-m}}{m n-m} d \gamma_{t}-\int_{0}^{\infty} \gamma_{t}^{m-1} e^{-a \gamma_{t}} \frac{\left(\frac{a \gamma_{t}}{z}\right)^{m n-m+1}}{m n-m+1} d \gamma_{t}\right. \\
& \left.+\int_{0}^{\infty} \gamma_{t}^{m-1} e^{-a \gamma_{t}} \frac{\left(\frac{a \gamma_{t}}{z}\right)^{m n-m+2}}{m n-m+2} d \gamma_{t}-\cdots+\int_{0}^{\infty} \gamma_{t}^{m-1} e^{-a \gamma_{t}} \frac{(-1)^{k}\left(\frac{a \gamma_{t}}{z}\right)^{m n-m+k}}{k !(m n-m+k)} d \gamma_{t}-\cdots\right] \\
& =\frac{n a^{m}}{\Gamma(m) \Gamma(m n-m)}\left[\frac{\Gamma(m n)}{(m n-m) a^{m} z^{m n-m}}-\frac{\Gamma(m n+1)}{(m n-m+1) a^{m} z^{m n-m+1}}\right. \\
& \left.+\frac{\Gamma(m n+2)}{2 !(m n-m+2) a^{m} z^{m n-m+2}}-\cdots+\frac{(-1)^{k} \Gamma(m n+k)}{k !(m n-m+k) a^{m} z^{m n-m+k}}-\cdots\right] \\
& =\frac{n}{\Gamma(m) \Gamma(m n-m)} \sum_{k=0}^{\infty} \frac{(-1)^{k} \Gamma(m n+k)}{k !(m n-m+k) z^{m n-m+k}}
\end{aligned}
$$

In fact, we can get the expression of capture probability in Rayleigh fading channel by Equation (30) with $m=1$. However, this equation, introducing infinite series, is not as simple as Equation (11).

\section{Optimal Frame Length and Maximum Channel Utilization}

In the FSA-based networks, we assume that the current frame length is $L$, which is divided into slots with equal duration. Also, we consider that $N$ users want to transmit packets and each randomly choosing one slot for transmission in the current frame. For a given slot, there are three probable outcomes: no packet transmitted (an idle slot), only one packet transmitted (a successful slot) and at least two packets transmitted (a collided slot). The procedure that $N$ packets select slots in frame length $L$ can be seen as $N$-fold Bernoulli trials with probability $\frac{1}{L}$. Therefore, the probability of $r$ packets transmitted in the same slot is given by

$$
p(r)=\left(\begin{array}{c}
N \\
r
\end{array}\right)\left(\frac{1}{L}\right)^{r}\left(1-\frac{1}{L}\right)^{N-r}
$$

where $\left(\begin{array}{c}N \\ r\end{array}\right)=\frac{N !}{r !(N-r) !}$. Then, the probabilities that a slot is an idle slot $(r=0)$, a successful slot $(r=1)$ or a collided slot $(r \geq 2)$ are $p_{i d l}, p_{s u c}$ and $p_{c o l}$, respectively, which can be given by

$$
\begin{aligned}
& p_{\text {idl }}=\left(1-\frac{1}{L}\right)^{N} \\
& p_{\text {suc }}=\frac{N}{L}\left(1-\frac{1}{L}\right)^{N-1} \\
& p_{\text {col }}=1-\left(1-\frac{1}{L}\right)^{N}-\frac{N}{L}\left(1-\frac{1}{L}\right)^{N-1}
\end{aligned}
$$

Therefore, when the capture effect occurs (A collided slot possibly becomes a successful slot), the expected number of idle, successful and collided slots are respectively given by 


$$
\begin{aligned}
& c_{i d l}=p_{i d l} L \\
& c_{\text {suc }}=p_{\text {suc }} L+L \sum_{i=2}^{i_{\max }} p_{\text {cap }}(n=i) p(r=i) \\
& c_{\text {col }}=L \sum_{i=2}^{i_{\max }}\left[1-p_{\text {cap }}(n=i)\right] p(r=i)
\end{aligned}
$$

where $p_{c a p}(\cdot)$ is the capture probability, which can be obtained from (1) on the basis of different values of $n$. In addition, $i_{\max }$ is the maximum number of packets colliding in the same slot. Then, the channel utilization can be defined by

$$
\eta=\frac{c_{s u c}}{L}=\frac{N}{L}\left(1-\frac{1}{L}\right)^{N-1}+\sum_{i=2}^{i_{\max }} \frac{N !}{i !(N-i) !}\left(\frac{1}{L}\right)^{i}\left(1-\frac{1}{L}\right)^{N-i} p_{\text {cap }}(n=i)
$$

Here, we take a linear model into account, i.e., $L=\lambda N$ with $0<\lambda \leq 1$. Then we can obtain

$$
\begin{aligned}
\lim _{N \rightarrow+\infty} \frac{N-1}{N} & =1 \\
\lim _{N \rightarrow+\infty}\left(1-\frac{1}{\lambda N}\right)^{N} & =e^{-\frac{1}{\lambda}}
\end{aligned}
$$

Substituting (39) and (40) into (38), the channel utilization is given by

$$
\eta \approx \frac{1}{\lambda} e^{-\frac{1}{\lambda}}+\sum_{i=2}^{i_{\max }} \frac{\lambda^{-i}}{i !} e^{-\frac{1}{\lambda}} p_{c a p}(n=i)
$$

Let (41) be the maximum, we can obtain

$$
\lambda^{*}=\arg \max _{0<\lambda \leq 1}\left[\frac{1}{\lambda} e^{-\frac{1}{\lambda}}+\sum_{i=2}^{i_{\max }} \frac{\lambda^{-i}}{i !} e^{-\frac{1}{\lambda}} p_{\text {cap }}(n=i)\right]
$$

Then, the optimal frame length can be calculated by

$$
L_{o p t}=\left\lceil\lambda^{*} N\right\rceil
$$

where $\lceil\cdot\rceil$ is a ceiling integer function. After substituting (43) into (38) or (41), the maximum channel utilization can be obtained.

For an intuitive explanation, we give one special case to show the capture effect impacts on the optimal frame length and the channel utilization. We assume the capture probability $p_{c a p}$ keeps unchanged in every collided slot of frame $L$ like literature [11] (note the reality is not like this). Then, the channel utilization can be rewritten as

$$
\begin{aligned}
\eta^{\prime}=\frac{c_{\text {suc }}^{\prime}}{L} & =\frac{p_{\text {suc }} L+p_{c a p} p_{c o l} L}{L} \\
& =\frac{N}{L}\left(1-\frac{1}{L}\right)^{N-1}+\left[1-\left(1-\frac{1}{L}\right)^{N}-\frac{N}{L}\left(1-\frac{1}{L}\right)^{N-1}\right] p_{c a p}
\end{aligned}
$$

In order to maximize the channel utilization, we take the first derivative of $\eta^{\prime}$ with respect to $L$ and let the derivative equals to zero, i.e.,

$$
\frac{d \eta^{\prime}}{d L}=\frac{N\left(p_{c a p}+N-N p_{c a p}-L\right)(L-1)^{N-2}}{L^{n+1}}=0
$$


For $L$ is an integer no less than one in reality, we can obtain the optimal frame length

$$
L_{\text {opt }}^{\prime}=\left(1-p_{\text {cap }}\right) N+p_{\text {cap }}
$$

Substituting (46) into (44), the maximum channel utilization can be written as

$$
\eta_{\text {max }}^{\prime}=\left[1-\frac{1}{\left(1-p_{\text {cap }}\right) N+p_{\text {cap }}}+p_{\text {cap }}\right]\left(1-p_{\text {cap }}\right)+p_{\text {cap }}
$$

According to (46), the optimal frame length is less than $N$ for $0<p_{\text {cap }}<1$ if the capture effect exists. As described in [21], the optimal frame length is equal to $N$ if the capture effect does't exist. In other words, the capture effect reduces the demand of slots for transmitting the same number of packets by making some of the collided slots to be successful slots and eventually shortens the optimal frame length. Finally, the channel utilization is improved by setting the optimal frame length.

\section{Numerical Results}

In this section, we give numerical results to manifest that the capture effect affects the channel utilization by MATLAB (version 8.3, The MathWorks, Natick, MA, USA) . In the experiments, we consider a scenario that there are $N$ active users transmitting packets to a base station and each transmits once in the frame length $L$. For example, Figure 1 gives the case of $N=8$, where each user randomly chooses a slot in the frame. Slot 1 , Slot 3, Slot 6 and Slot $L$ are all successful slots. Slot 4 and Slot $L-1$ are collided slots, and maybe they can turn into successful slots when the capture effect occurs. The others are all idle slots, which are not chosen by any users.

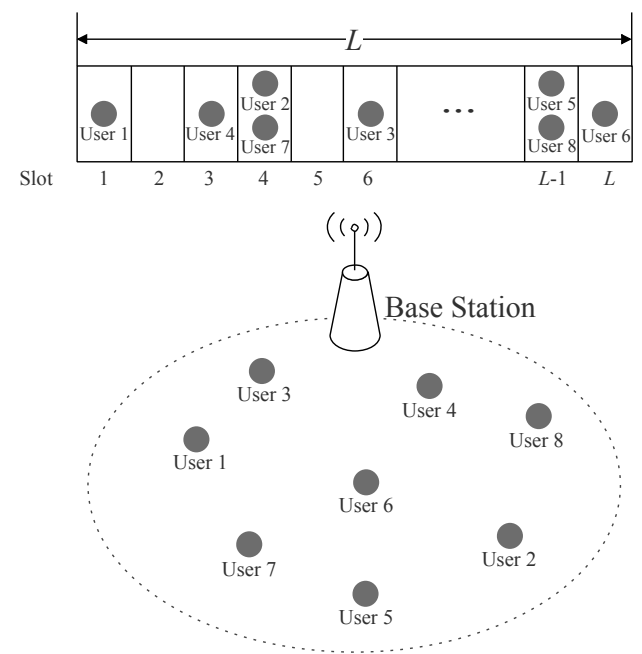

Figure 1. Each user randomly chooses a slot in the frame of length $L$ when the number of active users $(N)=8$.

\subsection{Capture Probabilities}

For showing the capture probabilities under Rayleigh, Rician and Nakagami- $m$ fading, we plot the analysis results in Figure 2a, Figure $2 b$ and Figure 2c according to (11), (22) and (30), respectively. In addition, we also give the simulation results in Figure 2, which accord with the analysis results. Besides, we give Table 1 for some special values of capture probabilities.

Figure 2a shows the capture probabilities with different values of capture ratio $z$ when the values of $n$ (the number of packets transmitted in the same slot) are 2, 3 and 4 in Rayleigh fading channel. As we can see from the figure and Table 1 , the capture probability decreases when the capture ratio $z$ increases. Furthermore, if $n$ increases, the capture probability decreases as well and decreases faster as a function of $n$ than $z$. 
According to (22), the capture probability in Rician fading channel depends on the factor $K$, the capture ratio $z$ and $n$. From Figure $2 \mathrm{~b}$ and Table 1 , the capture probability decreases when $z$ or $n$ increases like that of Rayleigh fading. In addition, the capture probability decreases as the Rician factor $K$ increases. Because larger $K$ means that the ratio of the power of LOS component to the average power of scattered components is higher, which results in a lower possibility that the condition for capture is satisfied.

Also, we plot the capture probabilities in Nakagami- $m$ fading channel based on (30), which are decided by the shape parameter $m$, the capture ratio $z$ and $n$. As seen in Figure $2 c$ and Table 1 , the capture probability decreases when $z$ or $n$ increases like that in Rayleigh and Rician fading. Moreover, the capture probability also decreases as the shape parameter $m$ increases. That is to say larger $m$ makes the condition for capture is more difficult to achieve.

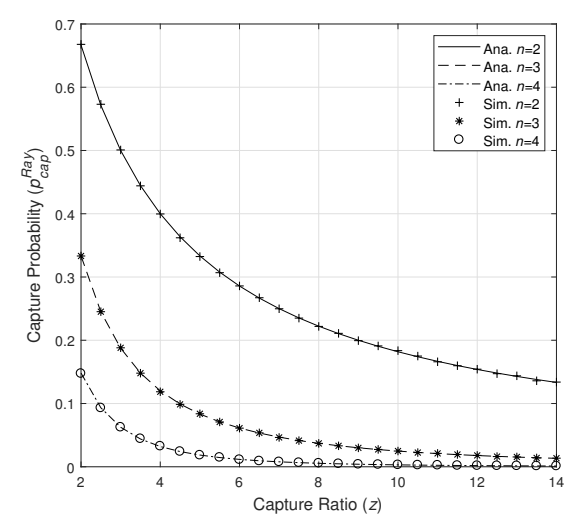

(a)

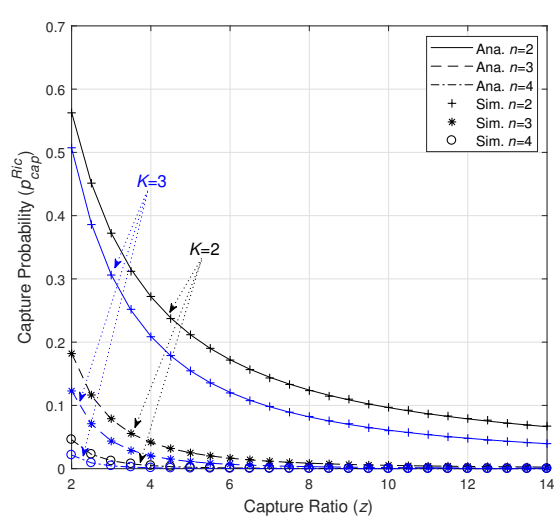

(b)

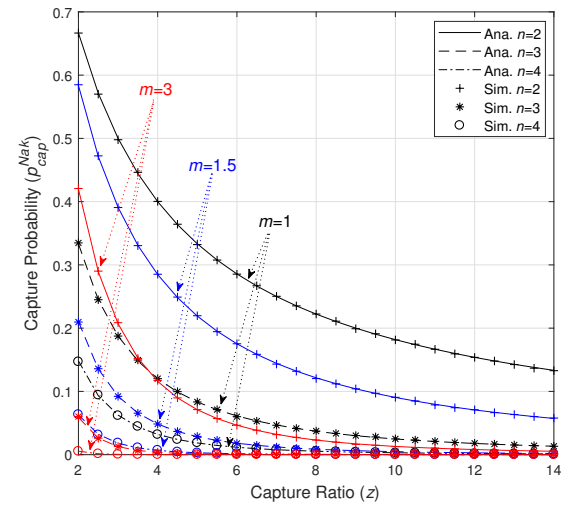

(c)

Figure 2. Capture (cap) probabilities when $n=2,3,4$. (a) Rayleigh (Ray) fading; (b) Rician (Ric) fading for $K=2,3$; (c) Nakagami- $m$ (Nak) fading for $m=1,1.5,3$. Ana., analysis; Sim., simulation.

Table 1. Capture probabilities under different fading channels for $z=2,4,10$. $(K=3, m=1.5)$.

\begin{tabular}{crrlrcccccc}
\hline \multirow{2}{*}{ Fading } & \multicolumn{3}{c}{$\boldsymbol{z}=\mathbf{2}$} & \multicolumn{3}{c}{$\boldsymbol{z}=\mathbf{4}$} & \multicolumn{3}{c}{$\boldsymbol{z}=\mathbf{1 0}$} \\
\cline { 2 - 10 } & $\boldsymbol{n}=\mathbf{2}$ & $\boldsymbol{n}=\mathbf{3}$ & $\boldsymbol{n}=\mathbf{4}$ & $\boldsymbol{n}=\mathbf{2}$ & $\boldsymbol{n}=\mathbf{3}$ & $\boldsymbol{n}=\mathbf{4}$ & $\boldsymbol{n}=\mathbf{2}$ & $\boldsymbol{n}=\mathbf{3}$ & $\boldsymbol{n}=\mathbf{4}$ \\
\hline Rayleigh & 0.667 & 0.333 & 0.148 & 0.400 & 0.120 & 0.032 & 0.182 & 0.025 & 0.003 \\
Rician & 0.506 & 0.123 & 0.022 & 0.209 & 0.020 & 0.001 & 0.061 & 0.002 & 0.000 \\
Nakagami- $m$ & 0.584 & 0.210 & 0.063 & 0.285 & 0.048 & 0.007 & 0.091 & 0.005 & 0.000 \\
\hline
\end{tabular}




\subsection{Optimal Frame Length}

In order to illustrate the probabilities that different number of packets collide in the same slot, we plot Figure 3, where the analysis results based on (31) are in line with the simulation results. In the figure, the collided probabilities of $r=2$ are higher than that of $r=3$ or 4 if the frame length $L$ is close to $N$. As described in [21], if the capture effect is ignored, the optimal frame length is equal to $N$. So we analyze the collided probabilities when $L=N$. For instance, if $L=N=60$, the probabilities of $r=2,3,4$ are $0.189,0.060$ and 0.013 , respectively. In fact, from Figure 3, the collided probabilities of the same value of $r$ are almost the same if $L=N$. Thus, we can know that the majority of collided slots result from two packets transmitted simultaneously when the frame length is optimal. In addition, according to Figure 2 and Table 1, we can know that the capture probabilities of $n=r=2,3,4$ decrease in sequence when the other parameters are the same. Particularly, when $n=4$, the capture probabilities of different fading channels are all less than 0.15 when the value of capture ratio $z$ is not less than 2, and these values gradually drop to zero as $z$ increases. In addition, when $n=3$ and $z=4$, the capture probabilities are very small too. Therefore, when $z$ is greater than 2 , the probability that a collided slot caused by three or more packets becomes a successful slot due to the capture effect is very small.

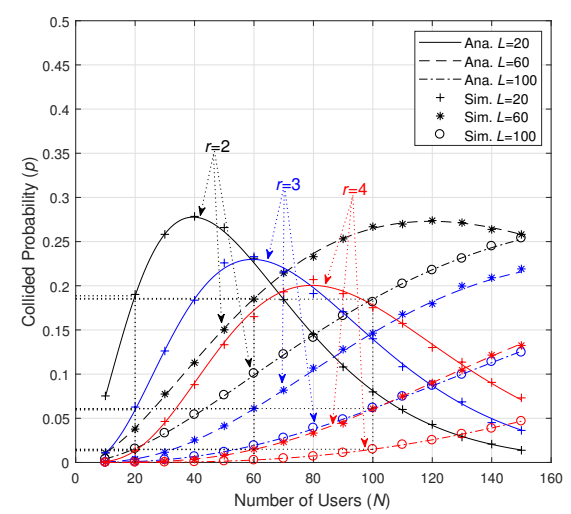

Figure 3. Collided probabilities when the number of packets transmitted in the same slot $(r)=2,3,4$.

From the above, as long as the frame length is optimal and $z$ is greater than two, that two packets collide in the same slot is more likely to take place, where the capture effect is more likely to occur than three or more packets collided. In this case, we can consider that the capture probability is the same in every collided slot of the frame. That is, all collided slots are caused by two packets.To achieve the maximum channel utilization, the optimal frame length can be set by (46). Let the Rician factor $K=3$, the Nakagami- $m$ shape parameter $m=1.5$, we can draw Figure 4a, which gives the optimal frame lengths about different values of $N$ based on (46) under Rayleigh, Rician and Nakagami- $m$ fading channels when $z=2,4,10$, respectively. In addition, we also give the optimal frame length with no capture effect $(L=N)$. From Figure $4 \mathrm{a}$, if the capture effect is taken into account, the optimal frame length is less than that of no capture effect. As $z$ increases, the optimal frame length becomes more close to that of no capture effect. The reason is that larger $z$ leads to smaller capture probability, which can be seen in Figure 2 and Table 1 . As a matter of fact, the capture probability can be close to zero if the value of $z$ is large enough. On this occasion, the optimal frame length is approximately equal to $N$.

However, if $z$ is not large, such as $z=2$, the capture probabilities of $n=3$ are all greater than 0.12 in Table 1, which cannot be ignored. Next, we take different capture probabilities of collided slots caused by different number of packets into account. Let $K=3, m=1.5$ and $z=2,4,10$, we plot Figure $4 \mathrm{~b}$, where the optimal frame length can be obtained by (42) and (43). From Figure $4 \mathrm{~b}$, we can find the optimal frame length is more close to $N$ as $z$ increases like that in Figure $4 a$, which is also caused by the capture effect. That is to say, fewer slots are needed to transmit the same number of packets 
when the capture effect exists. Compare Figure $4 a$ with Figure $4 b$, we find that the curves of the same value $z$ in the former are more far from the curve of no capture effect than that in the latter. Because Figure $4 \mathrm{a}$ is based on (46), which factitiously magnifies the real capture probability for assuming all collided slots are caused by two packets.

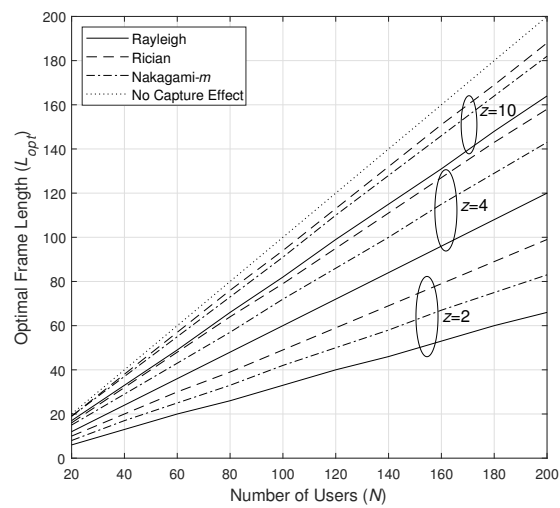

(a)

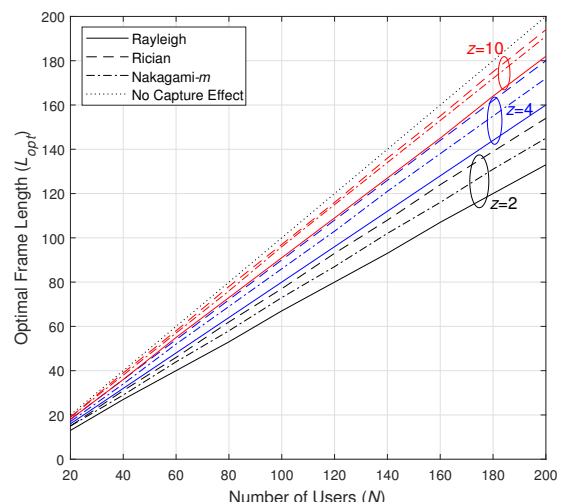

(b)

Figure 4. Optimal frame length when the capture ratio $(z)=2,4,10$. (a) Assume all the collided slots are only caused by two packets; (b) Collided slots are caused by two or more packets in practice.

\subsection{Channel Utilization}

In order to show the capture effect impacts on the channel utilization, we plot Figure 5a based on (47) first, where the simulation results are also showed. Here, we assume that the number of packets transmitted by the users in the current frame keeps unchanged, i.e., $N=100$. The other parameters are set as Figure 4a in Section 4.2, which implies that the collided slots are only caused by two packets. As seen in Figure 5a, which is one special case of literature [11], the maximum channel utilization with considering the capture effect is larger than 0.37 with no capture effect. In fact, if the durations of idle slot, successful slot and collided slot are not the same, the maximum channel utilization can also be larger than 0.37 even the capture effect is ignored [34]. Besides, the channel utilization decreases as capture ratio $z$ increases. The reason is that $z$ affects the capture probability. In addition, according to (38) and (47), we know that the capture probability affects the channel utilization. As a result, $z$ affects the channel utilization indirectly.

The above results are based on the contrived assumption that the collided slots are all caused by two packets. However, if $z$ is not large, such as $z=2$, the capture probabilities of $n=3$ are all greater than 0.12 in Table 1, which can not be ignored. In fact, the majority of collided slots are caused by two packets while the others are caused by three or more packets even the frame length is optimal. Next, we take different capture probabilities of slots caused by different number of packets into account. Then, the analysis results of channel utilization can be obtained by (38) or (41). For the sake of simplicity, we just give the analysis results and simulation results of channel utilization for $z=2,4,10$ when $N=100$, and the maximum values of channel utilization are specifically given in Table 2. From Figure 5b, the curves of different fading become more and more close to that of no capture effect as $z$ increases. The reason is as described in the previous paragraph. In a word, the capture effect can improve the channel utilization, and if we set the optimal frame length by (42) and (43), the maximum channel utilization can be obtained. 


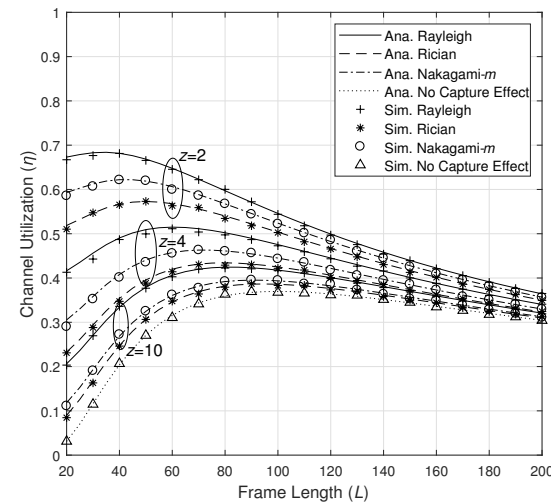

(a)

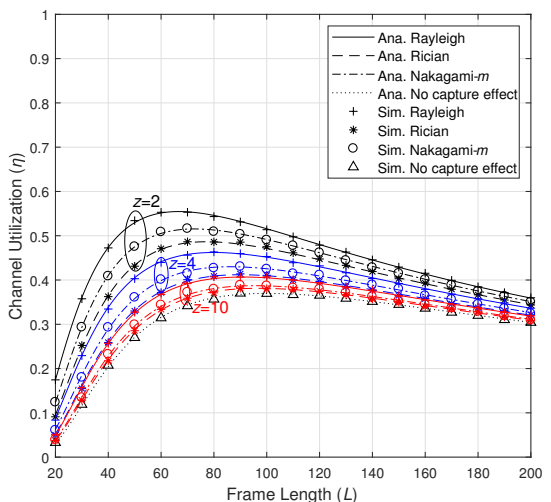

(b)

Figure 5. Channel Utilization when $z=2,4,10$. (a) Assume all the collided slots are only caused by two packets; (b) Collided slots are caused by two or more packets in practice.

Table 2. Theoretical maximum channel utilization.

\begin{tabular}{cccc}
\hline Fading & $z=\mathbf{2}$ & $\boldsymbol{z}=\mathbf{4}$ & $\boldsymbol{z}=\mathbf{1 0}$ \\
\hline Rayleigh & 0.56 & 0.46 & 0.41 \\
Rician & 0.49 & 0.41 & 0.38 \\
Nakagami- $m$ & 0.52 & 0.43 & 0.39 \\
\hline
\end{tabular}

Compare Figure $5 a$ with Figure $5 b$, we find that the maximum channel utilization of the same value $z$ in the former is greater than that in the latter. Because compared with the capture probability in the latter, the larger capture probability in the former leads to more collided slots turning into successful ones, which results in shortening the optimal frame length.

In order to verify the frame lengths of Figure $4 \mathrm{~b}$ are optimal, we plot the actual channel utilization versus different values of $N$ in Figure 6. For persuasive experimental results, Monte Carlo simulation method is adopted. From Figure 6, we can see that the actual channel utilization can be maximized in different fading channels by setting the optimal frame length. Actually, the values of actual channel utilization are all greater than 0.37 of no capture effect and approximately equal to the theoretical values in Table 2, respectively. In addition, the channel utilization decreases as $z$ increases. Because smaller $z$ leads to larger capture probability, which results in more collided slots turning into successful slots and eventually improves the channel utilization.

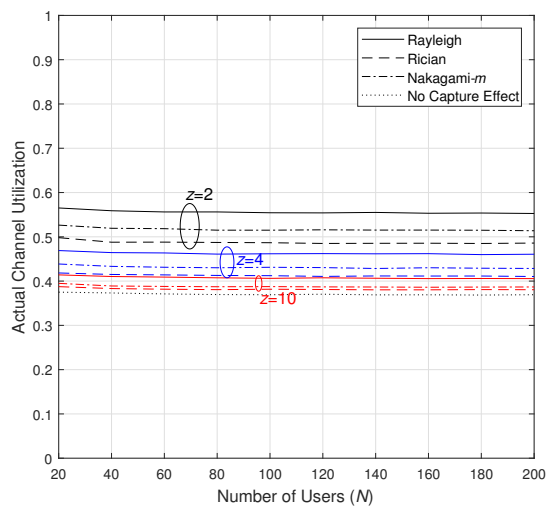

Figure 6. Actual channel utilization when $z=2,4,10$. 


\section{Conclusions}

In this paper, the capture effect under Rayleigh, Rician and Nakagami- $m$ fading channels has been analyzed and the closed-form expressions of capture probabilities are derived respectively. Furthermore, we give the optimal frame length which maximizes the channel utilization in the FSA-based networks. With the existence of capture effect, the maximum channel utilization of the FSA-based networks increases significantly, especially when capture ratio $z$ is small. In particular, we give a special case that the capture probability is the same in every collided slot and derive the optimal frame length. In addition, we also give the optimal frame length when the capture probability is changed by different number of collided packets in the slot. The numeral results show that the capture effect impacts on the channel utilization in the FSA-based networks under different fading channels. As a result, if the capture effect is efficiently utilized, the maximum channel utilization can be achieved by setting the optimal frame length.

Acknowledgments: The authors acknowledge the support from the Natural Science Foundation of China (Grant No. 61601388).

Author Contributions: Yang Wang researched the literatures, derived the formulas, carried out the simulations, and took charge of the entire manuscripts. Jianghong Shi revised the manuscript and verified the formulas. Lingyu Chen analyzed the simulation results and assisted with the integrity of the entire study.

Conflicts of Interest: The authors declare no conflict of interest.

\section{References}

1. Namislo, C. Analysis of mobile radio slotted ALOHA networks. IEEE J. Sel. Areas Commun. 1984, 2, 583-588.

2. Ma, R.T.; Misra, V.; Rubenstein, D. An analysis of generalized slotted-Aloha protocols. IEEE/ACM Trans. Netw. 2009, 17, 936-949.

3. Tseng, S.M.; Lin, H.P.; Chen, C.H.; Wang, Y.C. Throughput analysis of DS CDMA/unslotted ALOHA wireless networks with fixed packet length in Rayleigh fading finite-state Markov channel model. Wirel. Pers. Commun. 2013, 71, 3091-3104.

4. Tseng, S.M.; Chiang, L.H.; Wang, Y.C. Throughput of coded DS CDMA/Unslotted ALOHA networks with variable length data traffic and two user classes in Rayleigh fading FSMC model. KSII Trans. Internet Inf. Syst. 2014, 8, 4324-4342.

5. Liu, M.Y.; Wang, T.L.; Tseng, S.M. Throughput performance analysis of asynchronous optical CDMA networks with channel load sensing protocol. IEEE Photonics J. 2017, 9, 1-13.

6. Geraniotis, E.; Soroushnejad, M.; Yang, W.B. A multi-access scheme for voice/data integration in hybrid satellite/terrestrial packet radio networks. IEEE Trans. Commun. 1995, 43, 1756-1767.

7. Casini, E.; De Gaudenzi, R.; Herrero, O.D.R. Contention resolution diversity slotted ALOHA (CRDSA): An enhanced random access scheme for satellite access packet networks. IEEE Trans. Wirel. Commun. 2007, 6, 1408-1419.

8. Su, J.; Sheng, Z.; Hong, D.; Wen, G. An effective frame breaking policy for dynamic framed slotted Aloha in RFID. IEEE Commun. Lett. 2016, 20, 692-695.

9. Li, B.; Wang, J. Efficient anti-collision algorithm utilizing the capture effect for ISO 18000-6C RFID protocol. IEEE Commun. Lett. 2011, 15, 352-354.

10. Salah, H.; Ahmed, H.A.; Robert, J.; Heuberger, A. A time and capture probability aware closed form frame slotted ALOHA frame length optimization. IEEE Commun. Lett. 2015, 19, 2009-2012.

11. Wang, Y.; Wu, H.; Zeng, Y. Capture-aware estimation for large-scale RFID tags identification. IEEE Signal Process. Lett. 2015, 22, 1274-1277.

12. Espes, D.; Lagrange, X.; Suarez, L. A cross-layer MAC and routing protocol based on slotted Aloha for wireless sensor networks. Ann. Telecommun. 2015, 70, 159-169.

13. Iannello, F.; Simeone, O.; Spagnolini, U. Medium access control protocols for wireless sensor networks with energy harvesting. IEEE Trans. Commun. 2012, 60, 1381-1389.

14. Ioo, Y.I.; Kim, S.T.; Kim, J.W. An efficient beacon scheduling scheme for multi-hop in vehicular environments. Wirel. Pers. Commun. 2015, 83, 1085-1099. 
15. Mao, Y.; Yan, F.; Shen, L. Multi-round elimination contention-based multi-channel MAC scheme for vehicular ad hoc networks. IET Commun. 2017, 11, 421-427.

16. Huang, J.; Li, Q.; Zhong, S.; Liu, L.; Zhong, P.; Wang, J.; Ye, J. Synthesizing existing CSMA and TDMA based MAC protocols for VANETs. Sensors 2017, 17, 338.

17. Madueno, G.C.; Pratas, N.K.; Stefanović, Č.; Popovski, P. Massive M2M access with reliability guarantees in LTE systems. In Proceedings of the 2015 IEEE International Conference on Communications (ICC), London, UK, 8-12 June 2015; pp. 2997-3002.

18. Bello, L.M.; Mitchell, P.; Grace, D. Application of Q-learning for RACH access to support M2M traffic over a cellular network. In Proceedings of the 2014 20th European Wireless Conference, Barcelona, Spain, 14-16 May 2014; pp. 1-6.

19. Durand, F.R.; Abrão, T. Energy efficient adaptive optical CDMA random access protocol based on particle swarm optimization. Photonic Netw. Commun. 2017, 33, 275-289.

20. Shoaie, M.A.; Khazraei, S.; Pakravan, M.R. Performance analysis of slotted ALOHA random access packet-switching optical CDMA networks using generalized optical orthogonal codes and M-ary overlapping PPM signaling. J. Opt. Commun. Netw. 2011, 3, 568-576.

21. Chen, W.T. An accurate tag estimate method for improving the performance of an RFID anticollision algorithm based on dynamic frame length ALOHA. IEEE Trans. Autom. Sci. Eng. 2009, 6, 9-15.

22. Yang, L.; Zhang, G.; Lin, F.; Zheng, H. An efficient estimation method coping with the capture effect for RFID tags identification and application in remote learning. J. Intell. Fuzzy Syst. 2016, 31, 2707-2712.

23. Jun, J.; Yeon, S.; Kundu, T.; Agrawal, D.P.; Jeong, J. Caca: Link-based channel allocation exploiting capture effect for channel reuse in wireless sensor networks. In Proceedings of the 2016 IEEE 36th International Conference on In Distributed Computing Systems (ICDCS), Nara, Japan, 27-30 June 2016; pp. 600-608.

24. Sheikh, A.U.; Yao, Y.D.; Wu, X. The ALOHA systems in shadowed mobile radio channels with slow or fast fading. IEEE Trans. Veh. Technol. 1990, 39, 289-298.

25. Sanchez-Garcia, J.; Smith, D.R. Capture probability in Rician fading channels with power control in the transmitters. IEEE Trans. Commun. 2002, 50, 1889-1891.

26. Da Silva, A.P.T.R.; Brito, J.M.C. Analysis of adaptive modulation performance in networks with multiple access Slotted Aloha. In Proceedings of the 2017 31st International Conference on In Information Networking (ICOIN), Da Nang, Vietnam, 11-13 January 2017; pp. 210-215.

27. Khoshnevis, B.; Khalaj, B.H. Optimum power selection algorithms in Aloha networks: Random and deterministic approaches. IEEE Trans. Wirel. Commun. 2007, 6, 3124-3136.

28. Hua, Y.; Wang, Y. On the saturate throughput of IEEE 802.11 DCF with capture effect in Rician fading channel. In Proceedings of the 2008 4th International Conference on Wireless Communications, Networking and Mobile Computing (WiCOM), Dalian, China, 19-21 September 2008; pp. 1-4.

29. Hadzi-Velkov, Z.; Spasenovski, B. Capture effect in IEEE 802.11 basic service area under influence of Rayleigh fading and near/far effect. In Proceedings of the 2002 13th IEEE International Symposium on Personal, Indoor and Mobile Radio Communications (PIMRC), Lisboa, Portugal, 18 September 2002; pp. 172-176.

30. Gradštejn, I.S.; Ryžik, I.M. Table of Integrals, Series, and Products; Elsevier/Academic: Amsterdam, The Netherlands, 2007.

31. Jameel, F.; Haider, M.A.A.; Butt, A.A. Performance analysis of VANETs under Rayleigh, Rician, Nakagami- $m$ and Weibull fading. In Proceedings of the 2017 IEEE International Conference on Communication, Computing and Digital Systems (C-CODE), Islamabad, Pakistan, 8-9 March 2017; pp. 127-132.

32. Abramowitz, M.; Stegun, I.A. Handbook of Mathematical Functions with Formulas, Graphs, and Mathematical Tables; Dover: New York, NY, USA, 1972.

33. Simon, M.K.; Alouini, M.S. Digital Communication over Fading Channels; John Wiley \& Sons: Hoboken, NJ, USA, 2005.

34. Chen, W.T. Optimal frame length analysis and an efficient anti-collision algorithm with early adjustment of frame length for RFID systems. IEEE Trans. Veh. Technol. 2016, 65, 3342-3348.

(C) 2018 by the authors. Licensee MDPI, Basel, Switzerland. This article is an open access article distributed under the terms and conditions of the Creative Commons Attribution (CC BY) license (http://creativecommons.org/licenses/by/4.0/). 